\title{
Experimental Investigation of IEEE802.11n Reception with Fractional Sampling
}

\author{
Ryosuke Nakamura, Mamiko Inamori, Yukitoshi Sanada \\ Department of Electronics and Electrical Engineering, Keio University \\ Yokohama, 223-8522 Japan \\ Email:rnakamura@snd.elec.keio.ac.jp, inamori@elec.keio.ac.jp, sanada@elec.keio.ac.jp
}

\begin{abstract}
In the IEEE802.11n WLAN standard, orthogonal frequency division multiplexing (OFDM) modulation is employed. Diversity techniques are implemented to overcome multipath fading in the OFDM systems. A fractional sampling (FS) scheme is one of the diversity techniques with a single antenna. This scheme can also be applied in a MIMO-OFDM system to increase its capacity. In this paper, the effect of FS in a WLAN system following the IEEE802.11n standard is investigated through experiments. Numerical results through the experiments indicate that diversity gain through FS can be obtained in the NLOS conditions.
\end{abstract}

\section{INTRODUCTION}

IEEE802.11n WLAN is one of broadband communication standards specified in 2009 [1]. In the IEEE802.11n WLAN standard, orthogonal frequency division multiplexing (OFDM) is adopted as a modulation scheme. This is because OFDM based systems can achieve high frequency utilization efficiency due to orthogonal subcarriers [2], [3].

A multiple-input multiple-output (MIMO) technique adopted in IEEE802.11n is also widely used in recent wireless communication standards to realize high-speed and reliable transmission [4]. The MIMO technique employs multiple antennas both at a transmitter and a receiver in order to increase the capacity of the MIMO system. Even though the capacity of the MIMO system can be increased with additional antennas, the form factor of the terminal may limit the number of antenna elements.

On the other hand, for an OFDM receiver, a fractional sampling (FS) scheme has been proposed to resolve this problem [5]. FS can obtain diversity gain with a single antenna by sampling the received signal higher than the baud rate. Experimental investigation of FS in the IEEE802.11a/g WLAN standard has already been carried out in [6]. However, its diversity effect has not been confirmed with the IEEE802.11n system. Therefore, in this thesis, the experimental investigation of FS with the IEEE802.11n signal is carried out. The improvement of the capacity with FS in the IEEE $802.11 \mathrm{n}$ system lies on the selection of the sampling point [7], [8]. In the conventional MIMO system, the timing synchronization is carried out in terms of the received signals sampled at the baud rate [9-11]. In timing synchronization, the synchronization point is selected with peak detection at the output of the correlator. In this thesis, in conjunction with FS, timing synchronization is conducted on the signal sampled higher than the baud rate. Through the selection of the synchronization point, path diversity is realized.

This paper is organized as follows. Firstly, the FS scheme is described briefly in Section II. An experiment system is then explained in Section III. Numerical results are shown in Section IV. Finally, conclusions are presented in Section V.

\section{OFDM WITH Fractional SAMPLING}

Suppose there are $M_{t}$ transmit antenna elements and $M_{r}$ receive antenna elements in a MIMO-OFDM system. The following expression focuses on the $m_{t}$ th transmit and $m_{r}$ th receive antenna elements.

The information symbol on the $k$ th subcarrier from the $m_{t}$ th antenna is $s_{m_{t}}[k](k=0, \ldots, N-1)$, and then the OFDM symbol is given as

$$
u_{m_{t}}[n]=\frac{1}{\sqrt{N}} \sum_{k=0}^{N-1} s_{m_{t}}[k] \exp \left(j \frac{2 \pi n k}{N}\right)
$$

where $n(n=0,1, \ldots, N-1)$ is the time index, and $N$ is the size of the inverse discrete Fourier transform (IDFT). After appending guard interval with the duration of $N_{G I}$, the transmitted signal in a baseband form is given by $x(t)=$ $\sum_{n=-N_{G I}}^{N-1} u_{m_{t}}[n] p_{\mathrm{tx}}\left(t-n T_{s}\right)$, where $p_{\mathrm{tx}}(t)$ is the impulse response of the transmit filter and $T_{s}$ is the symbol duration. This signal is up-converted and transmitted through a multipath channel between the $m_{t}$ th transmit antenna and the $m_{r}$ th receive antenna with an impulse response, $c_{m_{r} m_{t}}(t)$. The received signal after down-conversion is given as

$$
y_{m_{r} m_{t}}(t)=\sum_{n=-N_{G I}}^{N-1} u_{m_{t}}[n] h_{m_{r} m_{t}}\left(t-n T_{s}\right)+v_{m_{r}}(t)
$$

where $h_{m_{r} m_{t}}(t)$ is the impulse response of the composite channel including the baseband filters on the $m_{t}$ th transmit antenna and the $m_{r}$ th receive antenna and is given by $h_{m_{r} m_{t}}(t):=p_{\mathrm{tx}}(t) \star c_{m_{r} m_{t}}(t) \star p_{\mathrm{rx}}(t)$, where $\star$ denotes convolution, $p_{\mathrm{rx}}(t)$ is the impulse response of the receive filter, and $v_{m_{r}}(t)$ is the noise on the $m_{r}$ th antenna. If $y_{m_{r} m_{t}}(t)$ is sampled at the rate of $T_{s} / G$, where $G$ is the oversampling 
rate, its polyphase components can be expressed as

$$
\begin{array}{r}
y_{m_{r} m_{t}}[n, g]=\sum_{l=-N_{G I}}^{N-1} u_{m_{r} m_{t}}[l] h_{m_{r} m_{t}}[n-l, g] \\
+v_{m_{r}}[n, g]
\end{array}
$$

where $g=\{0, \ldots, G-1\}, y_{m_{r} m_{t}}[n, g], h_{m_{r} m_{t}}[n, g]$, and $v_{m_{r}}[n, g]$ are the polynomials of sampled $y_{m_{r} m_{t}}(t), h_{m_{r} m_{t}}(t)$, and $v_{m_{r}}(t)$, respectively, and are expressed as

$$
\begin{aligned}
y_{m_{r} m_{t}}[n, g] & :=y_{m_{r} m_{t}}\left(n T_{s}+g T_{s} / G\right), \\
h_{m_{r} m_{t}}[n, g] & :=h_{m_{r} m_{t}}\left(n T_{s}+g T_{s} / G\right), \\
v_{m_{r}}[n, g] & :=v_{m_{r}}\left(n T_{s}+g T_{s} / G\right) .
\end{aligned}
$$

After removing the guard interval (GI) and taking discrete Fourier transform (DFT), the received symbol on the $k$ th subcarrier is given by

$$
\mathbf{z}_{m_{r} m_{t}}[k]=\mathbf{H}_{m_{r} m_{t}}[k] s[k]+\mathbf{w}_{m_{r}}[k]
$$

where $\mathbf{z}_{m_{r} m_{t}}[k]=\left[z_{m_{r} m_{t}}[k, 0] \ldots z_{m_{r} m_{t}}[k, G-1]\right]^{T}, \mathbf{w}[k]=$ $\left[w_{m_{r}}[k, 0] \ldots w_{m_{r}}[k, G-1]\right]^{T}$, and $\mathbf{H}_{m_{r} m_{t}}[k]=\left[H_{m_{r} m_{t}}[k, 0]\right.$ $\left.\ldots H_{m_{r} m_{t}}[k, G-1]\right]^{T}$ are $G \times 1$ column vectors. The $g$ th component of the received symbol is given as $\left[\mathbf{z}_{m_{r} m_{t}}[k]\right]_{g}:=$ $z_{m_{r} m_{t}}[k, g]=\sum_{n=0}^{N-1} y_{m_{r} m_{t}}[n, g] e^{-\frac{j \frac{2 \pi k n}{N}}{N}}$ and similarly for $w_{m_{r} m_{t}}[k, g]$ and $v_{m_{r}}[k, g]$. The channel response of the $g$ th sample on the $k$ th subcarrier is given as $H_{m_{r} m_{t}}[k, g]=$ $\sum_{n=0}^{N-1} h_{m_{r} m_{t}}[n, g] \exp \left(-j \frac{2 \pi k n}{N}\right)$.

\section{EXPERIMENT SYSTEM}

\section{A. Measurement Setup}

1) Measurement Environment: Figure 1 shows the measurement environment. The room is shielded from electrical magnetic waves. The boxes and the shelves are made of steel. Through the measurement, non-LOS (NLOS) conditions are evaluated. The receiver is placed and the signal is received on 9 different positions with $6.14 \mathrm{~cm}$ (=wavelength) separation for each measurement point. Figure 2 illustrates the

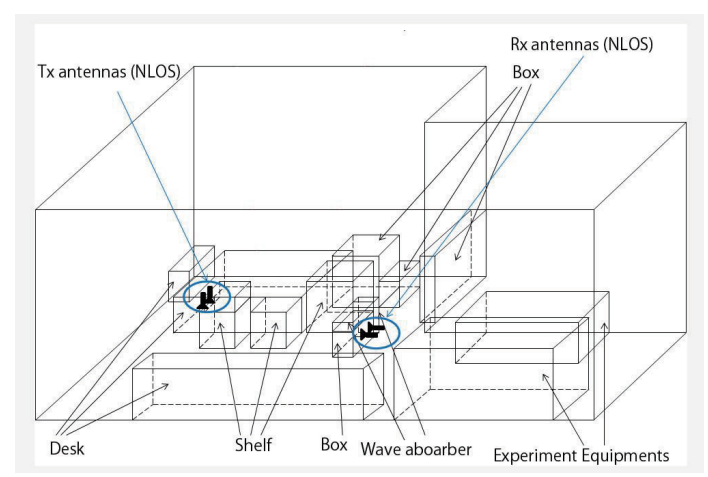

Fig. 1. Measurement room.

block diagram of the experiment system. Table 2 shows the specifications of the measurement equipments. The OFDM signals of the IEEE $802.11 \mathrm{n}$ system are generated by the signal

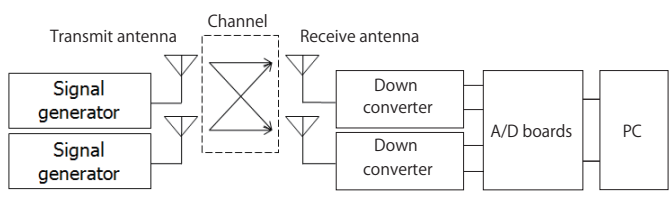

Fig. 2. Experiment system.

TABLE I

MEASUREMENT EQUIPMENTS

\begin{tabular}{|c|c|}
\hline $\begin{array}{c}\text { AD boards } \\
\text { Signatec } \\
\text { "PDA1000" }\end{array}$ & $\begin{array}{c}\text { Sampling frequency: } 250 \mathrm{MHz} \\
\text { Resolution: } 8 \text { bits }\end{array}$ \\
\hline $\begin{array}{c}\text { Down converter } \\
\text { Koden Electronics Co. } \\
\text { "A16-82100" }\end{array}$ & $\begin{array}{c}\text { Bandwidth of the baseband filter: } 40 \mathrm{MHz} \\
\text { Gain: }+40 \mathrm{~dB}\end{array}$ \\
\hline $\begin{array}{c}\text { ESG vector signal generator } \\
\text { Agilent } \\
\text { "E4438C" }\end{array}$ & $\begin{array}{c}\text { Frequency: from } 250 \mathrm{kHz} \text { to } 6 \mathrm{GHz} \\
\text { Maximum output power: }+17 \mathrm{dBm}\end{array}$ \\
\hline
\end{tabular}

generators. The received signals are down-converted to the baseband with the down-converter. In the down-converter, the bandwidth of the baseband filter is set to $40 \mathrm{MHz}$. The gain of the LNA is set to $+40 \mathrm{~dB}$. The outputs of the down-converter are digitized and saved in the $\mathrm{A} / \mathrm{D}$ boards with the sampling rate of $250 \mathrm{MHz}$ and the resolution of 8 bits. The sampling period of the received signal is $0.5 \mathrm{sec} / \mathrm{measurement}$.

2) Transmit Signal: In this experiment, the OFDM signal of the IEEE802.11n system is generated by the vector signal generator. Specifications of the OFDM signal used for the experiment are shown in Table II [1].

TABLE II SPECIFICATION OF SIGNAL

\begin{tabular}{|c|c|}
\hline Bandwidth of the channel & $20 \mathrm{MHz}$ \\
\hline Center frequency of the channel & $2.442 \mathrm{GHz}$ \\
\hline Modulation scheme & OFDM \\
\hline Number of subcarriers & 64 \\
\hline Number of data subcarriers & 56 \\
\hline Symbol duration & $3.2 \mu \mathrm{s}$ \\
\hline Guard interval & $0.8 \mu \mathrm{s}$ \\
\hline Number of transmit antennas & 2 \\
\hline Number of receive antennas & 2 \\
\hline
\end{tabular}

In this experiment, only the high throughput long training field (HT-LTF) is used as a transmit signal. When the number of transmit antennas is 2 , the number of the HT-LTF per packet is 2. The set of two HT-LTFs are transmitted repeatedly.

The OFDM signal is generated with the clock of $20 \mathrm{MHz}$ and is upsampled to $100 \mathrm{MHz}$. After upsampling, the signal passes through a transmit filter. This frequency response satisfies the IEEE802.11n spectrum mask. The frequency response of the filter is shown in Fig. 3. Finally, the filtered signal is transmitted from the signal generator.

\section{B. Signal Processing in Receiver}

The block diagram of the receiver used in this experiment is shown in Fig. 4. The received signal is down-converted to baseband and sampled. The DC offset of the sampled signal is removed and sampling rate conversion (SRC) is carried out. 


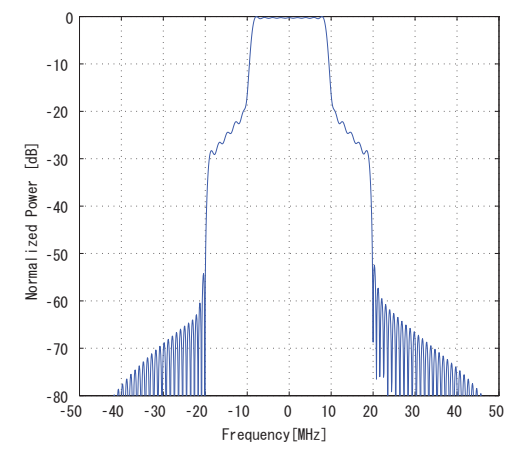

Fig. 3. Frequency response of transmit filter.

The sample rate after SRC is two times of the bandwidth of the desired signal, i.e. $40 \mathrm{MHz}$. Timing synchronization is achieved with the outputs of the correlators for the preamble sequence. Frequency offset is then removed and the calculation of correlation or the MIMO signals are demodulated. The

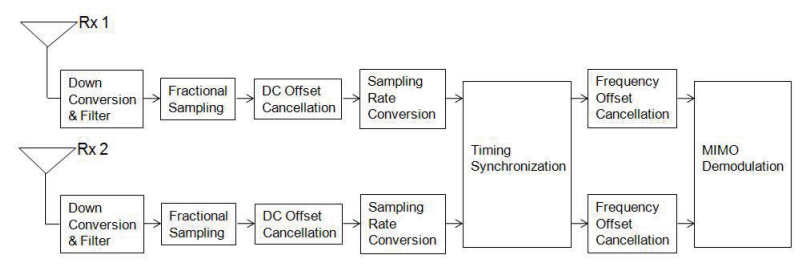

Fig. 4. Block diagram of signal processing in receiver.

demodulation process in each block is explained as follows.

1) DC Offset Cancellation: The DC offset of the received signal is estimated with the following equation.

$$
\hat{o}=\frac{\sum_{n=1}^{N_{a v}} y_{m_{r}}[n]}{N}
$$

where $y_{m_{r}}[n]$ is the $n$th sample of the received signal at the $m_{r}$ th antenna and $N_{a v}$ is the number of the samples for averaging. Averaging is carried out over each measurement period and $N_{a v}$ is about $5 \times 10^{6}$. DC offset cancellation can be achieved by subtracting $\hat{o}$ from the received samples as follows.

$$
\hat{y}_{m_{r}}[n]=y_{m_{r}}[n]-\hat{o}
$$

where $\hat{y}_{m_{r}}[n]$ is the $n$th sample at the $m_{r}$ th antenna of the receiver after DC offset cancellation.

2) Sampling Rate Conversion: SRC is carried out by the $M$ sets direct insertion/cancellation scheme in [12]. The $n$th sample after $M$ sets $(Q-\Gamma) / Q$ SRC is given as

$$
\tilde{y}_{m_{r}}[n]=\sum_{c=1}^{M} \hat{y}_{m_{r}}\left(\left\lceil\frac{n Q-\Gamma R_{c}+1}{Q-\Gamma}\right\rceil\right),
$$

where $\lceil\chi\rceil$ indicates the minimum integer number which exceeds $\chi$ and $R_{c}$ is a parameter which specifies the locations of the inserted or cancelled samples, which is expressed as

$$
R_{c}=\left\lceil\frac{c-1}{M}(Q-1)+\phi\right\rceil, \phi<\frac{Q-1}{M} .
$$

In this experiment, 25 set $24 / 25 \operatorname{SRC}(M=25, Q=24$ and $\Gamma=1$ ) is carried out.

3) Symbol Synchronization and Fractional Sampling: Before symbol synchronization, the received signal is downsampled with the ratio of $G / 12$ as

$$
\bar{y}_{m_{r}}[n]=\tilde{y}_{m_{r}}\left[\frac{12}{G} n\right] .
$$

This downsampling corresponds to $G$ times oversampling of the desired signal. The initial phase of the symbol is detected in the synchronization. Two schemes are used for synchronization. The block diagrams of the synchronization process are illustrated in Figs. 5 and 6 . The output of the

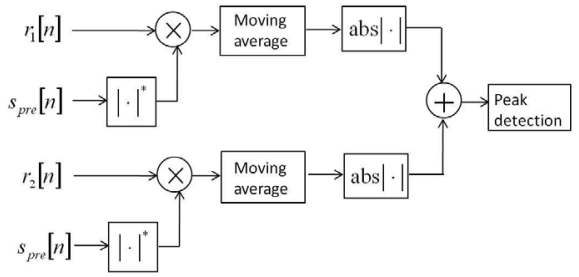

Fig. 5. Synchronization Scheme 1.

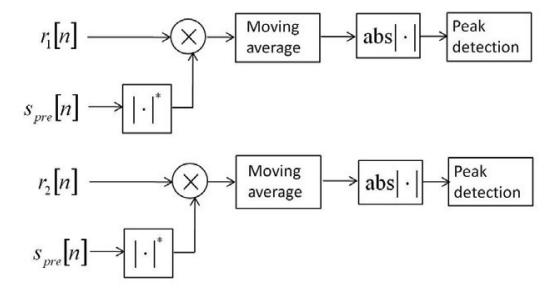

Fig. 6. Synchronization Scheme 2

combiner in Synchronization Scheme 1 (SS1) is given with the following equation

$$
\begin{array}{r}
O[n]=\sum_{\substack{m_{r}=1 \\
M_{r}}} \mid \sum_{m=1}^{N} \sum_{l=1}^{N_{L}} \bar{y}_{m_{r}}[(n+m-1+(l-1) \\
\left.\left.\left(N+N_{G I}\right)\right) G\right] \times s_{L}[n+m-1+N]^{*} \mid,
\end{array}
$$

where $s_{L}[n]$ is the $n$th sample of the HT-LTF, $N$ is the OFDM symbol duration, $N_{G I}$ is the length of the GI and $N_{L}$ is the number of the samples in the HT-LTFs. The $M_{r}$ outputs of the correlators are combined and put into the peak detector in this scheme. The synchronization points are the same on both of the antenna branches.

The output of the correlator in Synchronization Scheme 2 (SS2) is expressed with the following equation

$$
\begin{array}{r}
O_{m_{r}}[n]=\sum_{m=1}^{N} \sum_{l=1}^{N_{L}} \bar{y}_{m_{r}}[(n+m-1+(l-1) \\
\left.\left.\left(N+N_{G I}\right)\right) G\right] \times s_{L}[n+m-1+N]^{*} .
\end{array}
$$


The output of the correlator at each antenna branch is directly input to the peak detector. The synchronization point may be different between the antenna branches.

The peak output of the correlator is detected with the threshold. The threshold is set from 0.6 to 0.8 of the maximum output of the correlator.

4) Frequency Offset Cancellation: The $m_{r}$ th received signal with the frequency offset can be expressed as

$$
\begin{array}{r}
\bar{y}_{m_{r}}[n]=\sum_{l=0}^{N-1} \sum_{m_{t}=1}^{M_{t}} u_{m_{t}}[l] h_{m_{r}, m_{t}}[n-l] \exp \left(j \frac{2 \pi(l \varepsilon)}{N}\right) \\
+\Delta o+w_{m_{r}}[n]
\end{array}
$$

where $u_{m_{t}}[n]=\frac{1}{\sqrt{N}} \sum_{k=0}^{N-1} s_{m_{t}}[k] \exp \left(j \frac{2 \pi n k}{N}\right), s_{m_{t}}[k]$ is the transmitted symbol on the $k$ th subcarrier from the $m_{t}$ th antenna, $w_{m_{r}}[n]$ is the noise component of $\bar{y}_{m_{r}}[n], \varepsilon$ is the frequency offset normalized by subcarrier separation and $\Delta o=o-\hat{o}$ ( $o$ is the DC offset in the received signal) is the residual DC offset after cancellation. The frequency offset is calculated with each HT-LTF. The frequency offset is estimated as follows.

$$
\begin{aligned}
& \varepsilon^{\prime}=\frac{1}{2 \pi} \arg ( \sum_{m=1}^{N_{L}} \sum_{n=0}^{N_{G I}-1} \bar{y}_{m_{r}}[n+(m-1) N] \\
&\left.\times \bar{y}_{m_{r}}^{*}[n+(m-1) N+N]\right)
\end{aligned}
$$

where the guard interval of the HT-LTF starts from $n=0$. The received signal after removing the frequency offset that is estimated by the HT-LTF is given as

$$
\check{y}_{m_{r}}[n]=\bar{y}_{m_{r}}[n] \exp \left(-j \frac{2 \pi \varepsilon^{\prime}}{N} n\right) .
$$

$\check{y}_{m_{r}}[n]$ is put into the DFT block for MIMO demodulation.

5) MIMO Channel Estimation and Equalization: The channel response is estimated with the HT-LTFs. In this experiment, two transmit antennas are used for MIMO transmission. Therefore, there are two HT-LTFs and both the HT-LTFs are used for channel estimation. The received signals on the $k$ th subcarrier are expressed as the following equations.

$$
\mathbf{z}[k]=\mathbf{H}[k] \mathbf{s}_{L}[k]+\mathbf{w}[k]
$$

where

$$
\begin{gathered}
\mathbf{z}[k]=\left[\begin{array}{cc}
z_{11}[k] & z_{12}[k] \\
z_{21}[k] & z_{22}[k]
\end{array}\right], \mathbf{H}[k]=\left[\begin{array}{ll}
h_{11}[k] & h_{12}[k] \\
h_{21}[k] & h_{22}[k]
\end{array}\right], \\
\mathbf{s}_{L}[k]=\left[\begin{array}{cc}
s_{L}[k] & -s_{L}[k] \\
s_{L}[k] & s_{L}[k]
\end{array}\right], \mathbf{w}[k]=\left[\begin{array}{ll}
w_{11}[k] & w_{12}[k] \\
w_{21}[k] & w_{22}[k]
\end{array}\right],
\end{gathered}
$$

$z_{m_{r} j}[k]$ is the $j$ th HT-LTF received at the $m_{r}$ th receive antenna and $w_{i m_{r}}[k]$ is the noise component in $z_{i m_{r}}[k]$. The channel estimation is carried out as

$$
\hat{\mathbf{H}}[k]=\mathbf{z}[k] \mathbf{s}_{L}^{-1}[k]=\mathbf{H}[k]+\mathbf{w}[k] \mathbf{s}_{L}^{-1}[k] .
$$

In this experiment, the signal-to-noise ratio (SNR) of the received signal was about $30 \mathrm{~dB}$ and it is assumed that the channel estimation is nearly ideal.
After the channel estimation noise is added to adjust the SNR. The signal after SNR adjustment on the $k$ th subcarrier is given as

$$
\mathbf{z}^{\prime}[k] \check{\mathbf{z}}[k]+\mathbf{w}_{a d d}[k]
$$

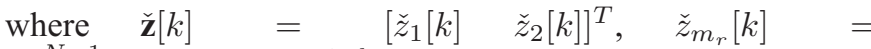
$\sum_{n=0}^{N-1} \check{y}_{m_{r}}[n] \exp \left(-j \frac{2 \pi k n}{N}\right)$, and $\mathbf{w}_{a d d}[k]$ is the added noise. After noise adjustment, the channel is equalized with Zero-Forcing and the BER is then measured. The estimated symbols after channel equalization are given as

$$
\begin{aligned}
\hat{\mathbf{s}}_{L}[k] & =\hat{\mathbf{H}}^{-1}[k] \mathbf{z}^{\prime}[k] \\
& =\hat{\mathbf{H}}^{-1} \mathbf{H}[k] \mathbf{s}_{L}[k]+\hat{\mathbf{H}}^{-1} \mathbf{w}^{\prime}[k]
\end{aligned}
$$

where $\mathbf{w}^{\prime}[k]$ is the noise component on the $k$ th subcarrier after SNR adjustment.

\section{NumERICAL RESUlts}

\section{A. Synchronization Performance}

Figure 7 is the synchronization probability with different correlator threshold levels. The SNR is set to $25 \mathrm{~dB}$. In the case of $G=1$, the synchronization probability decreases when the threshold increases while it is close to 1 with $G=2$. SS1 achieves better synchronization probability than SS2. Figure 8 shows the synchronization probability versus SNR with the threshold level is 0.6. If the SNR decreases, the synchronization probability improves with $G=2$ as compared to $G=1$. Moreover, at the low SNR region, the synchronization probability of SS1 is better than that of SS2. Even though the common synchronization point is not optimum in terms of the BER performance as shown in the following section, the summation of the correlator outputs brings more signal energy in the peak detection.

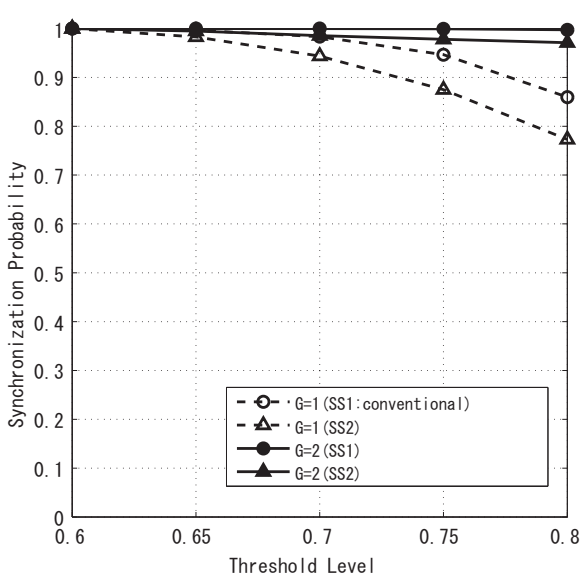

Fig. 7. Synchronization probability in the NLOS condition with different threshold levels $(\mathrm{SNR}=25 \mathrm{~dB})$. 


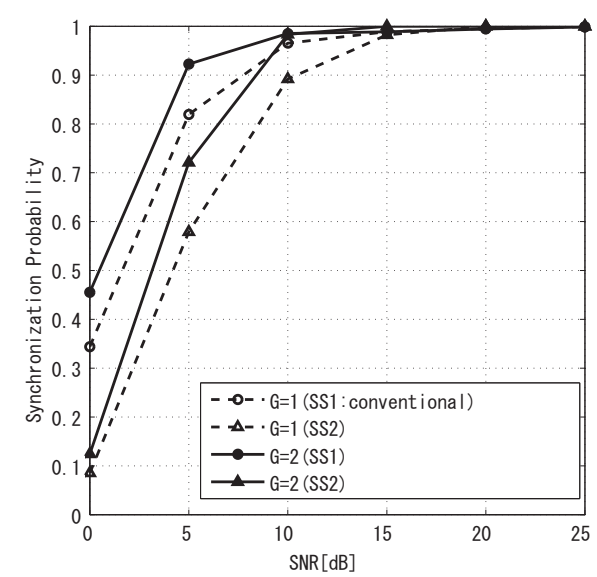

Fig. 8. Synchronization probability in the NLOS condition with different SNRs (threshold level=0.6).

\section{B. BER Performance}

Figure 9 shows the BER curve. The BER is calculated only when synchronization of the received signal is achieved. $E_{b} / N_{0}$ is equal to the SNR on each subcarrier because the modulation scheme is BPSK. These BER performance is calculated with the correlator threshold of 0.6.

In Fig. 9, the BER curve with $G=2$ shows about $0.5 \sim 1$ $\mathrm{dB}$ improvement as compared to that with $G=1$ for SS1 and about $2 \sim 2.5 \mathrm{~dB}$ improvement at the BER of $10^{-4}$ for SS2. This is because the multipath component is available and the path diversity is achieved.

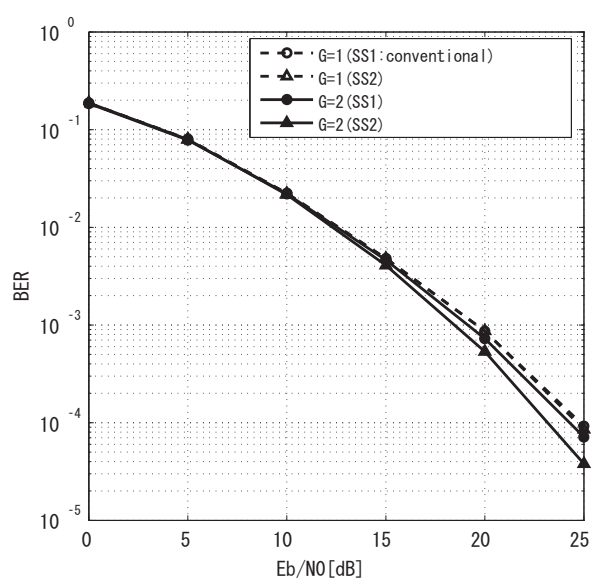

Fig. 9. BER performance in the NLOS condition (threshold level=0.6).

\section{CONCLUSions}

In this thesis, the effect of the FS scheme in the IEEE 802.11n WLAN system has been investigated through the experiment. In the FS receiver with $G$ times oversampled signals, two types of the synchronization schemes has been evaluated. One of them determines the common synchronization point for both of the antenna branches while the independent synchronization points are selected for each antenna branch in the other scheme. Numerical results showes that the oversampling and the independent sampling point selection has improved the performance by about $2 \sim 2.5 \mathrm{~dB}$ at the BER of $10^{-4}$ in the NLOS condition. It has also been clarified that oversampling improves the synchronization probability in the NLOS conditions. Consequently, it has been proven through the experiment that diversity gain in the FS scheme can be obtained in the IEEE 802.11n WLAN system in the NLOS condition.

\section{ACKNOWLEDGMENTS}

The authors acknowledge and express appreciations to Dr. Takeda at Toshiba Corporate Research \& Development Center for his support in the experiment. This work is supported in part by a Grant-in-Aid for the Global Center of Excellence for high-Level Global Cooperation for Leading-Edge Platform on Access Spaces and Grant-in-Aid for Scientific Research (C) under Grant No.22560390 from the Ministry of Education, Culture, Sport, Science, and Technology in Japan.

\section{REFERENCES}

[1] IEEE Std P802.11n/D11.0, Jun. 2009.

[2] B. Chen and H. Wang, "Blind Estimation of OFDM Carrier Frequency Offset via Oversampling," IEEE Trans. on Signal Processing, vol. 52, no. 7, pp. 2047-2057, July 2004

[3] J. Wang, J. Song, Z. X. Yang, L. Yang, and J. Wang, "Frames Theoretic Analysis of Zero-Padding OFDM Over Deep Fading Wireless Channels," IEEE Trans. on Broadcasting, vol. 52, no. 2, pp. 252-260, June 2006.

[4] H. Bölckei, D. Gesbert, and A.J. Paulraj, "On the Capacity of the OFDM-Based Spatial Multiplexing Systems," IEEE Trans. on Communications, vol. 50, no. 2, pp. 225-234, February 2002.

[5] C. Tepedelenlioĝlu and R. Challagulla, "Low-Complexity Multipath Diversity Through Fractional Sampling in OFDM," IEEE Trans. on Signal Processing, vol.52, no.11, Nov. 2004.

[6] T. Shinkai, H. Nishimura, M. Inamori, and Y. Sanada, "Experimental Investigation of Fractional Sampling in IEEE802.11a WLAN System," in Proc. the 11th IEEE International Conference on Communications Systems, pp. 1368-1373, Nov. 2008.

[7] H. Higuchi, H. Nishimura, and Y. Sanada, "Antenna/Sampling Point Selection Algorithm in Fractional Sampling-MIMO-OFDM," in Proc. the 11th IEEE International Conference on Communications Systems, pp. 411-416, Nov. 2008.

[8] K. Saito, T. Shinkai, H. Nishimura, and Y. Sanada, "Experiment Investigation of Sampling Point Selection in Fractional Sampling OFDM Receiver," in Proc. 2009 IEEE Pacific Rim Conference on Communications, Computers and Signal Processing, pp. 820-825, Aug. 2009.

[9] J. Cho, H. T. Anh, J. Kim and W. K. Cho, "Architecture of Timing Synchronization for MIMO-OFDM WLAN Systems," IEEE International Conference on Circuits and Systems for Communications 2008, pp. 210214, May. 2008

[10] M. Cho, Y. Jung and J. Kim, "Symbol Timing Synchronization for IEEE 802.11n WLAN Systems," First Asian Himalayas International Conference on Internet 2009, pp. 1-6, Nov. 2009.

[11] E. Zhou, X. Hou, J. Chen, Z. Zhang and H. Kayama, "FPGA Implementation and Experimental Performances of a Novel Timing Synchronization Method in MIMO-OFDM Systems," the 14th Asia-Pacific Conference on Communications 2008, pp. 1-5, Oct. 2008.

[12] A. B. Bostaman, Y. Sanada , and H. Minami, "Modified Direct Insertion/Cancellation Method Based Sample Rate Conversion for Software Defined Radio,” IEICE Trans. Commun., Vol.E91-B, No.8, Aug. 2008. 